\title{
Flip Angle
}

National Cancer Institute

\section{Source}

National Cancer Institute. Flip Angle. NCI Thesaurus. Code C73792.

In magnetic resonance imaging, the rotation of the average axis of the protons, relative to the main magnetic field direction, induced by radiofrequency signals. 